\title{
Computation of Supersonic Jet Mixing Noise for an Axisymmetric CD Nozzle Using k- $\varepsilon$ Turbulence Model
}

Abbas Khavaran

Sverdrup Technology, Inc.

Lewis Research Center Group

Brook Park, Ohio

and

Eugene A. Krejsa and Chan M. Kim

National Aeronautics and Space Administration

Lewis Research Center

Cleveland, Ohio

Prepared for the

30th Aerospace Sciences Meeting and Exhibit

sponsored by the American Institute of Aeronautics and Astronautics

Reno, Nevada, January 6-9, 1992

(NASA-TM-105338) COMPUTATION OF SUPERSONIC

N9 2-14795

JET MIXING NOISE FOR AN AXISYMMETRIC CD

NOZZLE USING K-EPSILON TURBULENCE MODEL

(NASA) $16 \mathrm{p}$

CSCL 2OA

$\begin{array}{ll} & \text { Unclas } \\ \mathrm{G3} / 71 & 0057142\end{array}$ 


\title{
Computation of Supersonic Jet Mixing Noise \\ for an Axisymmetric CD Nozzle Using k- $\epsilon$ Turbulence Model
}

\author{
Abbas Khavaran \\ Sverdrup Technology, Inc. \\ Lewis Research Center Group \\ Brook Park, Ohio $\mathbf{4 4 1 4 2}$
}

\author{
Eugene A. Krejsa* and Chan M. Kim \\ National Aeronautics and Space Administration \\ Lewis Research Center \\ Cleveland, Ohio 44135
}

\begin{abstract}
The turbulent mixing noise of a supersonic jet is calculated for a round convergent-divergent nozzle at the design pressure ratio. Aerodynamic computations are performed using the PARC code with a k- $\epsilon$ turbulence model. Lighthill's acoustic analogy combined with Ribner's assumption is adopted. The acoustics solution is based upon the methodology followed by GE in the MGB code. The source correlation function is expressed as a linear combination of second-order tensors. Assuming separable second-order correlations and incorporating Batchelor's isotropic turbulence model, the source term was calculated from the kinetic energy of turbulence. A Gaussian distribution for the time-delay of correlation was introduced. The CFD solution was used to obtain the source strength as well as the characteristic time-delay of correlation. The effect of sound/flow interaction was incorporated using the high frequency asymptotic solution to Lilley's equation for axisymmetric geometries. Acoustic results include sound pressure level directivity and spectra at different polar angles. The aerodynamic and acoustic results demonstrate favorable agreement with experimental data.
\end{abstract}

\section{Introduction}

Recent advances in the area of computational fluid dynamics have provided researchers with new tools for approaching aeroacoustic problems. Though the direct computation of the sound field and its propagation mechanism is the ultimate goal in an area which has been labeled as computational aeroacoustics, the extraordinary difficult nature of these computations at the present time lead to a more practical approach, namely using the available computational resources.

\footnotetext{
* Member, AIAA.
}

Such an approach is taken here to study the high speed jet noise because of the current interest in High Speed Civil Transport (HSCT).

Over a decade ago, a unified aerodynamic/acoustic prediction model was developed by GE for the Federal Aviation Administration [2]. The aerodynamic predictions within this code are carried out by applying an extension of Reichardt's model. The three compo nents of turbulent shearing stress are computed based upon contour integrals around the nozzle exit geometry and utilized in deriving an expression for the source strength. Though the closed form nature of Reichardt's solution has led to a versatile and relatively fast numerical scheme, the assumptions inherent in the derivations render inaccurate aerodynamic prediction for complex geometries.

Reichardt's solution essentially assumes exhaust nozzle elements discharge axially. It neglects radial mean flow and swirl and does not take into account the effect of shock structure on mixing and turbulence. These assumptions, among others, could vitiate the aerodynamic predictions substantially. In the light of these assumptions the model proves insufficient for noise prediction of new lobed hypermix nozzles that are the present focus in HSCT research. To circumvent some of the pitfalls associated with Reichardt's model, a two-stage algorithm is considered in which the aerodynamic calculations are carried out independently and the resulting plume data are introduced for noise computation. The following computational advantages are associated with a two-stage algorithm:

(1) the aerodynamic calculations can start from within the nozzle, giving a more realistic model of the exit conditions;

(2) grid selection can be done independently for aerodynamic and acoustic purposes. The plume is treated as an integration of axial slices in acoustic computations;

(3) proper prediction of shock-cell structure and intensity for off-design conditions improves shock noise assessment. 
The geometry considered in this paper is a round convergent-divergent nozzle. This particular nozzle has previously been tested aerodynamically and acoustically for NASA-Lewis by General Electric [1]. Test data has been especially helpful in assessing the CFD results before proceeding with noise computations. The flowpath is designed to obtain an isentropic, uniform and parallel flow at the nozzle exit for the design Mach number of 1.4 , thereby eliminating to a large degree, any shock induced noise. An axisymmetric version of PARC code with Chien's k- $\epsilon$ turbulence model [3] has been used. The complete Reynolds-averaged Navier Stokes equations are solved in conservative law form. The viscus coefficients are determined based on Sutherland's viscosity law, Stokes hypothesis and constant Prandtl number assumption. The jet plume flow field is compared with data as well as solution from Reichardt's model.

\section{Method of Solution}

The solution technique is essentially based upon the methodology followed by GE in developing the MGB code [2]. Reichardt's aerodynamic model is replaced with CFD and the technique is briefly outlined here with emphasis on the new changes and comparisons with the original model.

The solution technique is described in two sections, source spectrum model and sound/flow interaction. The first section describes the application of PARC with a $k-\epsilon$ turbulence model to computation of source strength and its spectrum and explains the empirical constants used in computation of supersonic convection factor. In the second section the effect of surrounding medium on radiated noise from convecting quadrupole sources is described. It is explained how the mean flow temperature and velocity, as obtained from $\overline{C F D}$, are used in computation of sound/flow interaction of axisymmetric jets.

\section{Source Spectrum Model}

The mean-square sound pressure in the far field, in absence of convection and refraction is written using Lighthill's acoustic analogy approach $[4,5]$.

$$
\overline{p^{2}}(R, \theta, \phi)=\frac{R_{i} R_{j} R_{k} R_{\ell}}{16 \pi^{2} C_{\infty}^{4} R^{6}} \int_{\vec{y}} \int_{\vec{\xi}} \frac{\partial^{4}}{\partial \tau^{4}} \overline{T_{i j} T_{k \ell}^{\prime}} d \vec{\xi} d \vec{y}
$$

The source strength is assumed to be dominated by unsteady momentum flux i.e. $T_{i j} \sim \rho V_{i} V_{j}$. Vector $\vec{\xi}$ in the above integration is the separation vector of the correlation between $\rho V_{i} V_{j}$ computed at $\vec{y}$ and $\rho^{\prime} V_{k}^{\prime} V_{\ell}^{\prime}$ at $\vec{y}+\vec{\xi}$ and $\tau$ is the time delay of correlation. The corresponding spectrum can be expressed in terms of the Fourier transform of the autocorrelation function

$$
\overline{p_{\omega}^{2}}=\frac{1}{2 \pi} \int_{-\infty}^{+\infty} \overline{p^{2}} e^{i \omega \tau} d \tau
$$

As such, for a quasi-incompressible turbulence, the source strength is characterized by a two-point timedelayed fourth-order velocity correlation tensor. For a nearly parallel mean flow, the constituent velocities may be written as the sum of the local mean velocity and the turbulent velocity, $V_{i}=U \delta_{i 1}+v_{i}$. Substituting $V_{i}$ into equation (1), the contribution to the integral due to the self noise terms is shown to be independent of the mean flow. This contribution, as shown by Ribner [6], is primarily due to terms like $\overline{v_{i} v_{j} v_{k}^{\prime} v_{l}^{\prime}}$. As explained by Mani [7], the distinction between shear noise and self noise becomes less important when the interpretation of the source terms is based upon Lilley's [8] formulation rather than Lighthill's equation. Assuming a normal joint probability for turbulent velocity components, as suggested by Ribner [6] and using the isotropic turbulence model of Batchelor [9], the fourth-order correlation can be written as a linear combination of second-order correlations.

$$
\overline{v_{i} v_{j} v_{k}^{\prime} v_{\ell}^{\prime}}=\left(\overline{v_{i} v_{k}^{\prime}}\right)\left(\overline{v_{j} v_{\ell}^{\prime}}\right)+\left(\overline{v_{i} v_{\ell}^{\prime}}\right)\left(\overline{v_{j} v_{k}^{\prime}}\right)+\left(\overline{v_{i} v_{j}^{\prime}}\right)\left(\overline{v_{k} v_{\ell}^{\prime}}\right)
$$

Further by assuming that the two-point velocity correlations, $\overline{v_{i} v_{j}^{\prime}}$, are separable in space/time factors, as postulated by Ribner,

$$
\overline{v_{i} v_{j}^{\prime}}=R_{i j}(\vec{\xi}) g(\tau)
$$

the integration on separation vector of correlation can be carried out in closed form. The space factor, according to Batchelor, is written as:

$$
R_{i j}(\vec{\xi})=T\left[\left(f+\frac{1}{2} \xi f^{\prime}\right) \delta_{i j}-\frac{1}{2} f^{\prime} \xi_{i} \xi_{j} / \xi\right]
$$

where $T=\frac{1}{3} \overline{v_{i} v_{i}}$ is the intensity of turbulence replacing the axial turbulence intensity in the Reichardt's model and $f^{\prime}=\partial f / \partial \xi$. The three components of the separation vector of correlation are represented as $\xi_{i}$ for $i=1,2,3$. Function $f(\xi)$ can be expressed as $f(\xi)=\exp \left(-\pi \xi^{2} / L^{2}\right)$. This expression makes $f(\xi)$ decrease to zero for large $\xi$ with sufficient rapidity to make $\int_{0}^{\infty} \xi^{m} f(\xi) d \xi$ converge for $m \geq 0$. Length $L$ is the longitudinal macroscale of turbulence, representing the linear extent of the region within which velocities are appreciably correlated. The time factor of correlation is expressed as $g(\tau)=\exp \left(-\tau / \tau_{0}\right)$ where $\tau_{0}$ denotes the characteristic time-delay in a moving reference frame. For axisymmetric jets, Davies et al. [10] have shown 
that $\tau_{0}$ is proportional to the inverse of mean shear i.e. $1 / \tau_{0}=(\partial U / \partial r)$ where $U$ denotes the mean velocity and $r$ the radial distance. In addition, turbulence intensity can be expressed in terms of $L$ and $\tau_{0}$ according to $\tau_{0} \sim L / \sqrt{T}$. Since the eddy length scale $L_{\epsilon}$ is related to kinetic energy of turbulence $k=\overline{v_{i} v_{i}} / 2$ and its dissipation rate $\epsilon$ as $L_{\epsilon}=k^{3 / 2} / \epsilon$, assuming that $L_{\epsilon}$ and $L$ are proportional, it can be concluded that $1 / \tau_{0} \sim \epsilon / k$. The above conclusion can also be derived from the algebraic turbulence model. Comparison of $r_{0}$, as computed from $k$ and $\epsilon$, with the corresponding results obtained from the velocity gradient and Reichardt's aerodynamic solution are presented under the numerical discussions.

Ribner [6] evaluates the contribution to self noise for various source strength components. The corresponding spectrum is evaluated by using a Fourier transform on the time delay of the correlation [2]. In the final analysis the source intensity spectrum can be expressed as

$$
I(\Omega) \sim \rho^{2} k^{\frac{\pi}{2}}\left(\Omega \tau_{0}\right)^{4} e^{\frac{-1}{\left(\Omega r_{0}\right)^{2}}}
$$

To compute the noise field, this equation will later be used in conjunction with the refraction effect of the mean flow. The doppler effect relating the source frequency $\Omega$ and the observed frequency $f$ is expressed through a convection factor, $\bar{C}$, as $\Omega=2 \pi f \bar{C}$. The singular behavior of the eddy convection factor $\bar{C}=$ $\left(1-M_{c} \cos \theta\right)$ at supersonic convection speeds $\left(M_{c}>1\right)$ has been discussed by Ffowcs Williams [11]. The autocorrelation function in this region, which corresponds to emission of eddy Mach wave, becomes zero and thus the integrand remains finite. In our analysis a modified convection factor described by

$$
\bar{C}=\sqrt{\left(1-M_{c} \cos \theta\right)^{2}+\left(\alpha_{c} k^{5} / C_{\infty}\right)^{2}}
$$

has been selected for numerical computation. The empirical convection constant $\alpha_{c}$ was determined from comparison of prediction with data. A value of $\alpha_{c}=.5$ is generally a good approximation for most observation angles. The convection Mach number was expressed as a function of the weighted average of the nozzle exit Mach number and local Mach $M$

$$
M_{c}=.5 M+\beta_{c} M_{j}
$$

Values in the range of .25 to .3 for the convection constant $\beta_{c}$ appear to yield best results.

\section{Sound/Flow Interaction}

Lighthill's acoustic analogy approach, based on classical wave equation doesn't incorporate the effect of surrounding mean flow on the sound radiation caused by convected multipole sources. It is clear that these pressure fluctuations should propagate through a region of nonuniform velocity and temperature before reaching the observer point. Thus the location of source within the jet plume has a strong effect on the amount of radiated sound. The mean flow results in not only the refraction of the radiated sound, but also an additional convection amplification due to fluid motion. A number of investigators have studied the radiation field of multipole sources immersed in parallel sheared flows. Mani $[7,12,13]$ studied the mean flow interaction of round jets for slug flow profiles assuming quadrupole sources convecting along the centerline of the jet. Gliebe and Balsa [14] and Goldstein [15] extended the above analysis to arbitrary velocity profiles in high frequency and low frequency limits respectively. The generalization to arbitrarily located sources in continuously varying monotonic profiles were derived by Balsa [16] and Goldstein $[17,18]$. For supersonic jets, the high frequency solution provides an adequate approximation as suggested by Tester and Morfey [19] and Pao [20].

Lilley's equation is considered as the starting point by most recent investigators studying the sound/flow interactions. For a parallel flow, the Green's function solution to a convected monopole of frequency $\Omega$ is obtained by applying a sequence of Fourier transformations. The solution corresponding to multipole singularities are obtained by differentiating the monopole solution with respect to appropriate source coordinate; once in the case of a dipole source and twice for quadrupole sources. Balsa and Mani [2] provide a comprehensive analysis of the shielding effect of an axisymmetric mean flow, i.e. when the jet velocity and temperature profiles are arbitrary functions of the radial variable. To study the effect of asymmetry in the mean flow, Goldstein [21] solves Lilley's equation for high frequency multipole sources in a jet flow whose Mach number and temperature are functions of crossflow coordinates. This leads to an ordinary differential equation that traces the cross-stream projection of an acoustic ray starting from any given source location. The differential equation is solved repeatedly as one selects initial departure angle for rays leaving the source. Subsequent ray tracing through the shear layer and study of the spreading or focusing of the acoustic emission as it approaches far field, results in a circumferential directivity factor for each source volume element.

For our axisymmetric geometry, Mani and Balsa's formulation, as used in the MGB code, has been applied whereby the turbulent properties of the jet has been coupled with its acoustic radiation. Thus the mean 
square pressure in the far field is expressed as

$$
\overline{p^{2}}(R, \theta, \Omega)=\int_{\bar{y}} \Lambda\left(a_{x x}+4 a_{x y}+2 a_{y y}+2 a_{y z}\right) d \vec{y}
$$

The contribution to noise field due to each of the quadrupoles contained within a turbulent eddy volume element is described by the directivity factors $a_{x x}, \ldots, a_{y z}$. The corresponding weighting factors were derived by Ribner [6]. $\Lambda$ is a factor related to the source intensity and frequency

$$
\Lambda \sim \frac{\left(\frac{\rho_{\infty}}{\rho}\right)^{2} I(\Omega)}{\left(4 \pi R C_{\infty} C\right)^{2}(1-M \cos \theta)^{2}\left(1-M_{c} \cos \theta\right)^{2}}
$$

The directivity factors are functions of the shielding function $g^{2}$ which is expressed in terms of the polar observation angle $\theta$, local sound velocity $C$ and Mach numbers $M(r)=U(r) / C_{\infty}$ and $M_{c}=U_{c} / C_{\infty}$.

$$
g^{2}(r)=\frac{(1-M)^{2}\left(\frac{C_{\infty}}{C}\right)^{2}-\cos ^{2} \theta}{\left(1-M_{c} \cos \theta\right)^{2}}
$$

The mean flow variables, as obtained from CFD, are used in computation of $g^{2}(r)$ and hence the directivity factors $a_{x x}, \ldots, a_{y z}$. Location $r$ where $g^{2}(r)$ changes in sign is called transition point or turning point. When $g^{2}(r)$ is negative between the source and observer, the possibility of fluid shielding exists. The position of the source with respect to the turning points of $g^{2}(r)$ contributes to the amount of fluid shielding through factor $\exp \left(-2 K \int_{r_{1}}^{r_{2}} \sqrt{\left|g^{2}(r)\right|} d r\right)$. The limits of the integration are determined by the location of the source relative to the turning points.

Wave number $K=\omega / C$ in the above exponential factor tends to increase the attenuation of the high frequencies. An extensive list of directivity factors corresponding to a case with two turning points have been listed in [2]. A maximum of four turning points have been considered in the present computations. A correction of one Doppler factor has been utilized for source volume effects according to Ffowcs Williams [11]. The correction for flight speed has been included using flight dynamic factor $\left(1+M_{\infty} \cos \theta\right)^{-1}$ where $M_{\infty}$ is the flight Mach number.

\section{Numerical Results and Discussion}

To obtain the flow field solution a $141 \times 60$ grid (Figure 1), highly clustered around the jet lip-line was used. A run time of over four hours on CRAYYMP was required to achieve the necessary convergence within PARC-ke code. The aerodynamic results obtained from PARC code were input to a noise prediction code. Aerodynamic and acoustic calculations were compared with available data. Also turbulent intensity and characteristic time delay of correlation, as predicted by PARC code, were compared with Reichardt's solution. All laser velocimeter, shadowgraph and acoustic tests for this model were conducted in the General Electric anechoic free-jet facility [1]. The test point (corresponding to the design condition) is listed in table 1. The Mach contour plot (Figure 2) indicates the formation of a Mach disc inside the nozzle as well as a weak shock outside the exit plane, also seen in the shadowgraph photo [2]:

Figures 3 and 4 demonstrate the close agreement with data in the velocity profiles on the lip-line, centerline, and also at four axial positions along the jet. The small oscillations in velocity near the centerline can better be predicted by increasing the grid resolution from exit to 10 diameters downstream. Figure 5 exhibits the contour plot for the intensity of turbulence, indicating the extension of noise sources to nearly $18 \mathrm{di}$ ameters downstream. The contour levels are nondimensionalized with respect to reference sound speed, $a^{2}{ }_{r}$, which is computed at the stagnation temperature. Further details of the turbulence intensity distribution are demonstrated in Figures 6 and 7. The axial distribution of the turbulence intensity near the lip-line (Figure 6a) shows a level of 13 percent near the exit plane with a growing decay rate after 12 diameters, generally in good agreement with data. Reichardt's model predicts a much faster decay after 5 diameters. The radial distribution (Figure $6 \mathrm{~b}$ ) was plotted only at $X / D=8.21$ where test data was available for comparison. Obviously PARC code does a better job in predicting the maximum turbulence level. Near the centerline, which is still within the potential core, the CFD computations clearly under-predict the data by 3 to 4 percent. This can be attributed to the boundary conditions used in a CFD solution and should not introduce any significant error in noise computation because of low level of source intensity and high shielding effect. Further comparison of the source term using the two aerodynamic prediction methods is illustrated in Figure 7. Reichardt's model predicts a faster spreading rate and also fails to properly predict the ending of the potential core where increased turbulence intensity near the centerline is expected. In addition, Reichardt's model does not accurately predict the axial decay of the turbulence intensity (see Figure 6a).

The characteristic time delay $\tau_{o}$ was obtained using two different definitions alluded to earlier. The corresponding Strouhal numbers defined as $(\epsilon / k)\left(D / U_{j}\right)$ and $(\partial U / \partial r)\left(D / U_{j}\right)$ are plotted in Figures 8 and 9 . It is clear that outside the proportionality factor $\alpha_{f}$ needed for the computation of $\tau_{0}$, these curves demonstrate remarkable resemblance, showing a peak value 
moving in toward the centerline with increasing $X$. A third curve was also included based on Reichardt's solution, showing the same general features but failing to properly predict the radial location of the maximum.

In our acoustic computations $\tau_{o}$ was calculated from $1 / \tau_{0}=\alpha_{f} \epsilon / k$. Factor $\alpha_{f}$ can shape the sound spectrum in terms of the location of the maximum and should be determined empirically. A value of $\alpha_{f}=2$ was selected in these calculations. For nonaxisymmetric geometries, additional modification in the definition of $\tau_{0}$ is necessary as the circumferential gradient of the mean velocity also contributes to this factor. The dissipation rate of turbulent kinetic energy is plotted in Figure 10. The contour levels are nondjmensionalized with respect to $a^{3}{ }_{r} / L_{r}$ where $L_{r}$ is the reference length used in the PARC code. The acoustic computations for a $\mathbf{4 0}$ foot radius are presented in Figures 11 and 12. The overall sound pressure (OASPL) directivity as obtained from PARC shows reasonable agreement with data at most observation angles. The empirical factors $\beta_{c}$ and $\alpha_{f}$ were selected to obtain the best fit at angles where the jet mixing noise is maximum. Obviously for off-design conditions, the shock noise dominates at angles close to upstream axis and several dB's difference in our predictions would not significantly alter the directivity of the total noise. The over-prediction of up $5 \mathrm{~dB}$ 's at $\theta=80$ degrees can be improved by selecting an angle dependent convection constant $\alpha_{c}$. Reichardt's solution also generates results in close agreement with data as the empirical constants used in this aerodynamic prediction model are calibrated for a conical flow field. The spectral components of noise, based on a one-third octave band are shown in figure 12. The overall agreement seems acceptable.

\section{Concluding Remarks}

The source strength has been successfully predicted using PARC code with a $k-\epsilon$ turbulence model. The resulting noise predictions compare reasonably with experimental data. This application has removed four empirical constants used in the Reichardt's model, which are calibrated for a conical flow field. The limitations on aerodynamic grid selection has been removed by adopting a two-stage aerodynamic and acoustic algorithm. Further, the time-delay of correlation has been computed using two different models. The acoustic computations, according to the present model, is based on two fundamental assumptions as suggested by Ribner and Batchelor. A proper prediction of the noise spectrum depends on an accurate assessment of the time-delay of correlation.

For non-axisymmetric geometries, a proper circumferential averaging of the flow variables can be used as a first step in the computation of the polar directivity of jet noise. Also contributions from other components of the mean velocity gradient should be included in the computation of $\tau_{0}$. More elaborate analysis such as geometric ray theory is needed to predict the azimuthal spreading of jet noise. In addition, the empirical constants introduced in the determination of supersonic convection factor should further be investigated for other geometries.

\section{Nomenclature}

\begin{tabular}{|c|c|}
\hline$C_{\overline{\bar{C}}} C_{\infty}$ & $\begin{array}{l}\text { local and ambient speeds of sound } \\
\text { convection factor }\end{array}$ \\
\hline$D, D_{\text {eq }}$ & exit and throat diameters \\
\hline$f$ & observed frequency \\
\hline$I(\Omega)$ & source intensity spectrum \\
\hline$k$ & turbulent kinetic energy $=\overline{v_{i} v_{i}} / 2$ \\
\hline$K$ & wave number \\
\hline$L$ & longitudinal macroscale of turbulence \\
\hline$L_{\epsilon}$ & eddy length scale \\
\hline$M, M_{j}$ & local and exit Mach numbers \\
\hline $\begin{array}{l}M_{c}, M_{\infty} \\
\quad r\end{array}$ & $\begin{array}{l}\text { convection and flight Mach numbers } \\
\text { radial distance }\end{array}$ \\
\hline$R$ & source to observer distance \\
\hline$R_{i}$ & observer coordinate \\
\hline$T$ & intensity of turbulence $=\overline{v_{i} v_{i}} / 3$ \\
\hline $\begin{array}{l}T_{i j} \\
U\end{array}$ & $\begin{array}{l}\text { unsteady momentum flux } \\
\text { mean velocity in direction of flow }\end{array}$ \\
\hline$U_{j}$ & exit jet velocity \\
\hline$v_{i}$ & $\begin{array}{l}\text { fluctuating velocity component in } X_{i} \\
\text { direction }\end{array}$ \\
\hline$V_{i}$ & $\begin{array}{l}\text { local velocity component in } X_{i} \\
\text { direction }\end{array}$ \\
\hline$\overline{v_{i} v_{j}^{\prime}}$ & two-points time-delayed second-order \\
\hline$\vec{y}$ & $\begin{array}{l}\text { correlation }=\int_{-\infty}^{+\infty} v_{i}(\vec{y}, t) v_{j}(\vec{y}+\vec{\xi}, t+\tau) d t \\
\text { source coordinate }\end{array}$ \\
\hline$\alpha_{c}, \beta_{c}$ & convection constants \\
\hline $\begin{array}{l}\alpha_{f} \\
\delta_{i j}\end{array}$ & $\begin{array}{l}\text { proportionality factor }\left(1 / \tau_{0}=\alpha_{f} \epsilon / k\right) \\
\text { Kronecker's delta }\end{array}$ \\
\hline$\epsilon$ & $\begin{array}{l}\text { dissipation rate of kinetic energy } \\
=\nu\left(\partial v_{i} / \partial X_{j}\right)\left(\partial v_{i} / \partial X_{j}\right)\end{array}$ \\
\hline$\theta$ & $\begin{array}{l}\text { polar angle with respect to } \\
\text { downstream axis }\end{array}$ \\
\hline$\underline{\Lambda}$ & source factor \\
\hline$\vec{\xi}$ & vector separation of correlation \\
\hline$\rho, \rho_{\infty}$ & local and ambient densities \\
\hline$\nu$ & molecular viscosity \\
\hline$\tau$ & time delay of correlation \\
\hline $\begin{array}{l}\tau_{0} \\
\phi\end{array}$ & $\begin{array}{l}\text { characteristic time delay of correlation } \\
\text { azimuthal angle }\end{array}$ \\
\hline$\omega, \Omega$ & observer and source radian frequencies \\
\hline
\end{tabular}

\section{References}


1. Yamamoto, h. J., et al, "Experimental Investigation of Shock-Cell Noise Reduction for Single Stream Nozzles in Simulated Flight - Comprehensive Data Report," NASA CR-168234, 1984.

2. Mani, R., et al, "High velocity Jet Noise Source Location and Reduction," Task 2, , FAA-RD-7679-II. 1977.

3. Chien, K. Y., "Prediction of Channel and Boundary layer Flows with a Low Reynolds-Number Turbulence Model," AIAA Journal, Vol. 20, Jan. 1982, pp. 33-38.

4. Lighthill, M. J., "On Sound Generated Aerodynamically. I General Theory," Proc. Roy. Soc. of London, Vol. A211, 1952, pp. 564-587.

5. Lighthill, M. J., "On Sound Generated derodynamically. II Turbulence as a Source of Sound," Proc. Roy. Soc. of London, Vol. A222, 1954, pp. $1-32$.

6. Ribner, H. S., "Quadrupole Corrections Governing the Pattern of Jet Noise," JFM, Vol 38. 1969, p. 1 .

7. Mani, R., "The Influence of Jet Flow on Jet Noise, Part I, The Noise of Unheated Jets," JFM, Vol. 76. 1976 , pp. $753-778$

8. Lilley, G. M.. Morris, P. J., and Tester, B. J., "On the theory of Jet Noise and its Application." AIAA paper No. 73-987, Oct. 1973.

9. Batchelor, G. K. The theory of Homogeneous Turbulence, Cambridge Univ. Press, 1953.

10. Davies, P. O. A. L., Fisher, M. J., and Barratt, II. J., "The Characteristics of the Turbulence in the Mixing Region of a Round Jet," JFM. Vol. 15, 1963. p. 337.

11. Ffowcs Williams, J. E., "The Noise from Turbulence Convected at High Speed," Phil. Trans. Roy. Soc. of London, Vol. A255, 1963. pp. 469 503.

12. Mani R. "Moving Source Problem Relevant to jet Noise," J. Sound and Vibration, Vol. 25, 1972, pp. $337-347$.

13. Mani, R. "The Influence of Jet Flow on the Jet Noise, Part II. The Noise of Heated Jets." JFM, Vol 76,1976 , p. 779 .

14. Gliebe, P. R., and Balsa, T. F., "The Aerodynamics and Acoustics of Coaxial Jet Noise," AIAA paper No. 76-492., Third Aeroacoustics Conference, 1976.
15. Goldstein. M. E., "The low Frequency Sound from Multipole Sources in Axisymmetric Shear Flows," $J F M$, Vol. 70, 1975, p. 595.

16. Balsa T. F., "The Acoustic Field of Sources in Shear Flow with Application to Jet Noise: Convective Amplification," $J F M$, Vol. 79, 1977, p. 33

17. Goldstein, M. E., "The low frequency Sound from Multipole Sources in Axisymmetric Shear Flows Part I," JFM, Vol. 75, 1976, p. 17.

18. Goldstein, M. E., Aeroacoustics, McGraw-Hill, 1976.

19. Tester, B. J. and Morfey, C. L., "Developments in Jet Noise Modeling - Theoretical Predictions and Comparisons with Measured Data," J. Sound and Vibration, Vol. 46(1), 1976, p. 79.

20. Pao, S. P., "Aerodynamic Noise Emission from Turbulent Shear Layers," JFM, Vol. 59, 1973, p. 451 .

21. Goldstein, M. E., "High Frequency Sound Emission from Moving Point Multipole Sources Embedded in Arbitrary Transversely Sheared Mean Flows," J. Sound and Vibration, Vol. 80(4), 1982, p. 499.

Table 1. Design Parmaters for C-D Nozzle

$\begin{array}{ll}\text { Throat Diameter } & 5.1 \mathrm{in} . \\ \text { Exit Diameter } & 5.395 \text { in. } \\ \text { Distance from Throat to Exit } & 5.525 \mathrm{in} \\ \text { Exit Velocity } & 2409 \mathrm{fps} \\ \text { Ambient Velocity } & 400 \mathrm{fps} \\ \text { Pressure Ratio } & 3.121 \\ \text { Stagnation Temp. } & 1716^{\circ} \mathrm{R}\end{array}$



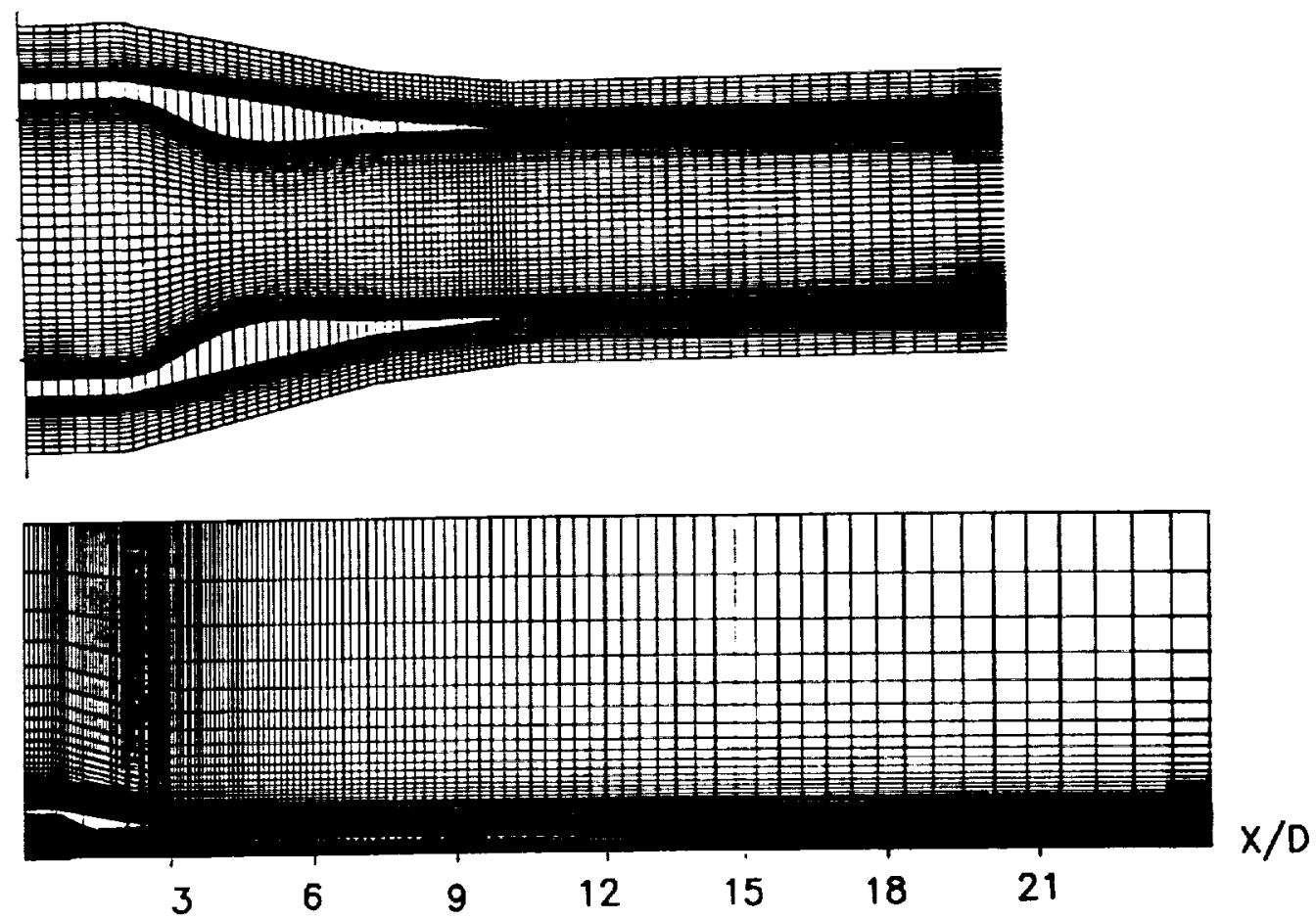

Fig. 1: Nozzle geometry and grid.

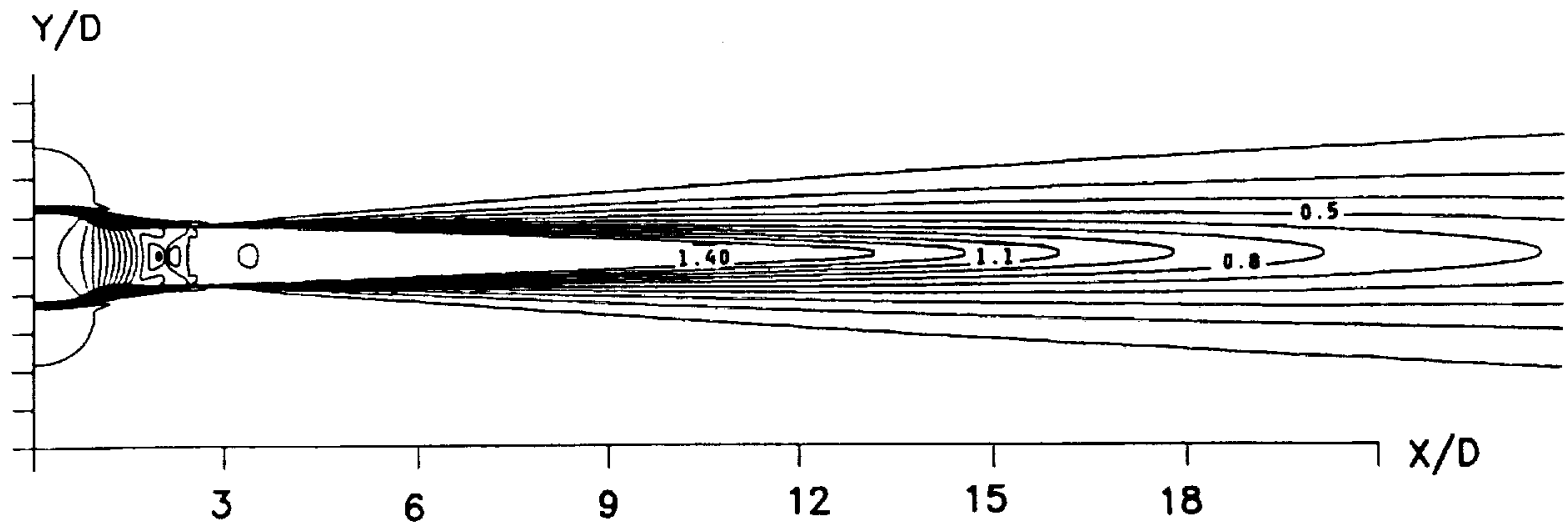

Fig. 2: Mach number contour plot $\left(M_{j}=1.4\right)$. 

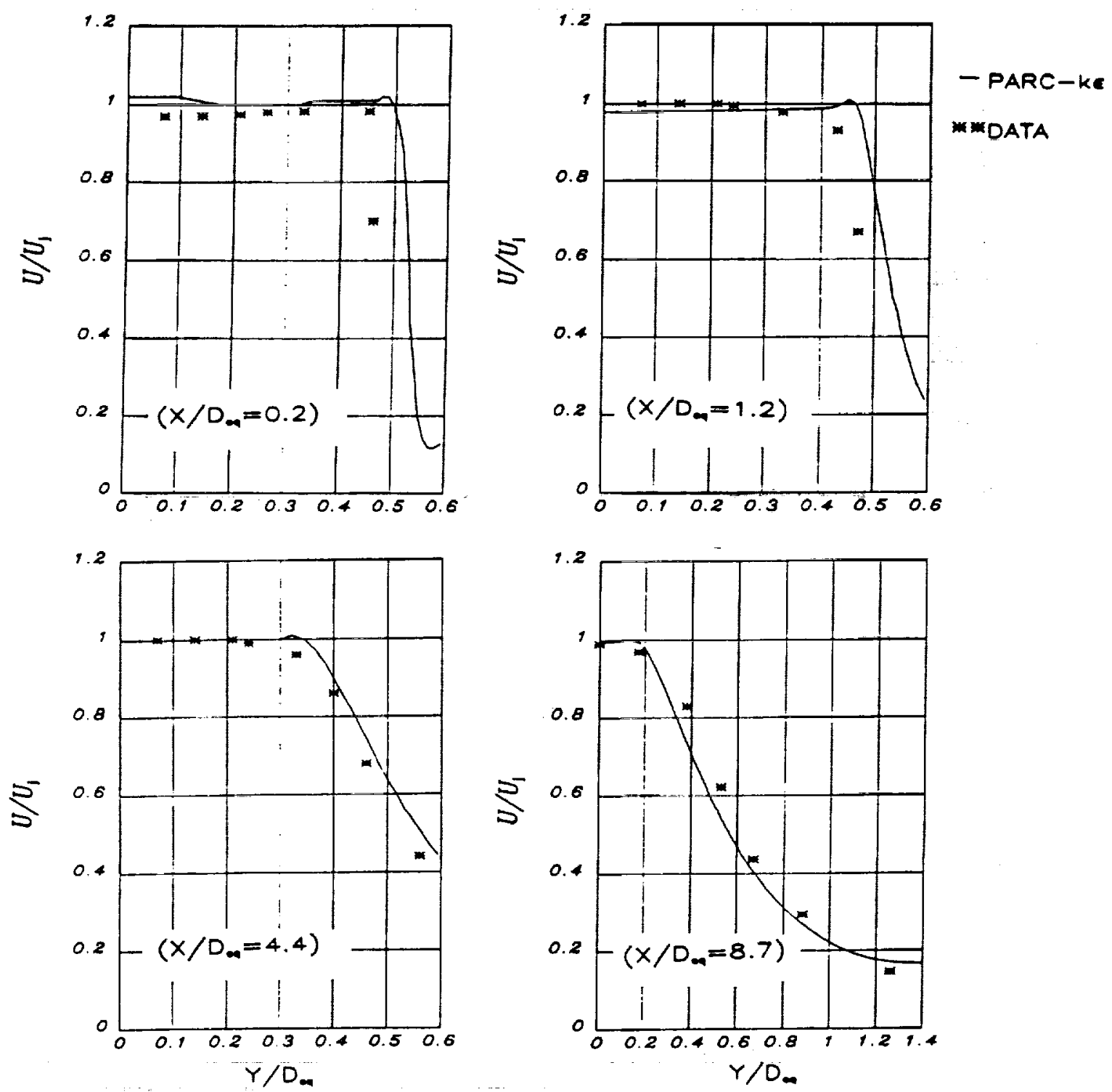

Fig. 3: Comparison of velocity profiles with experimental results [1] at four different axial locations. 

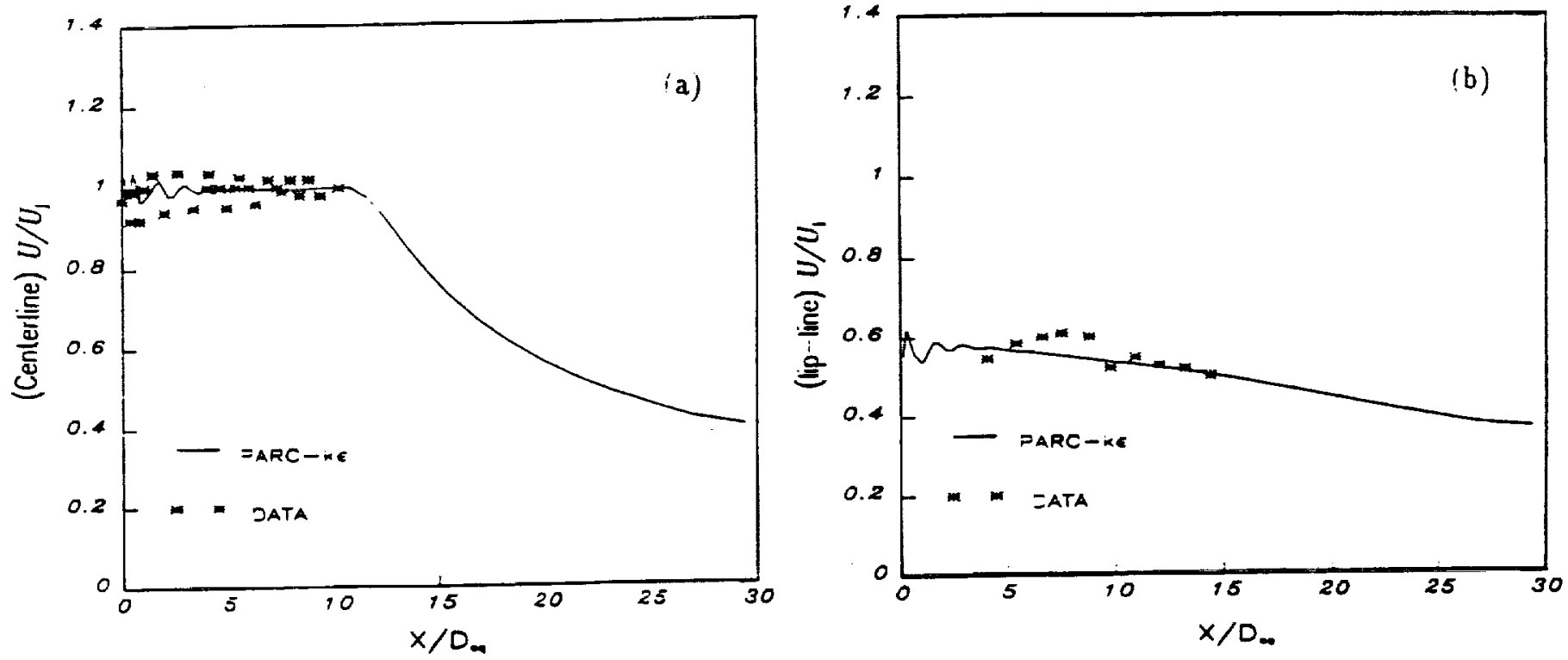

Fig. 4: Comparison of the reiocity profiles with experimental results [1] on the (a) cent eriine (b) lip-line.

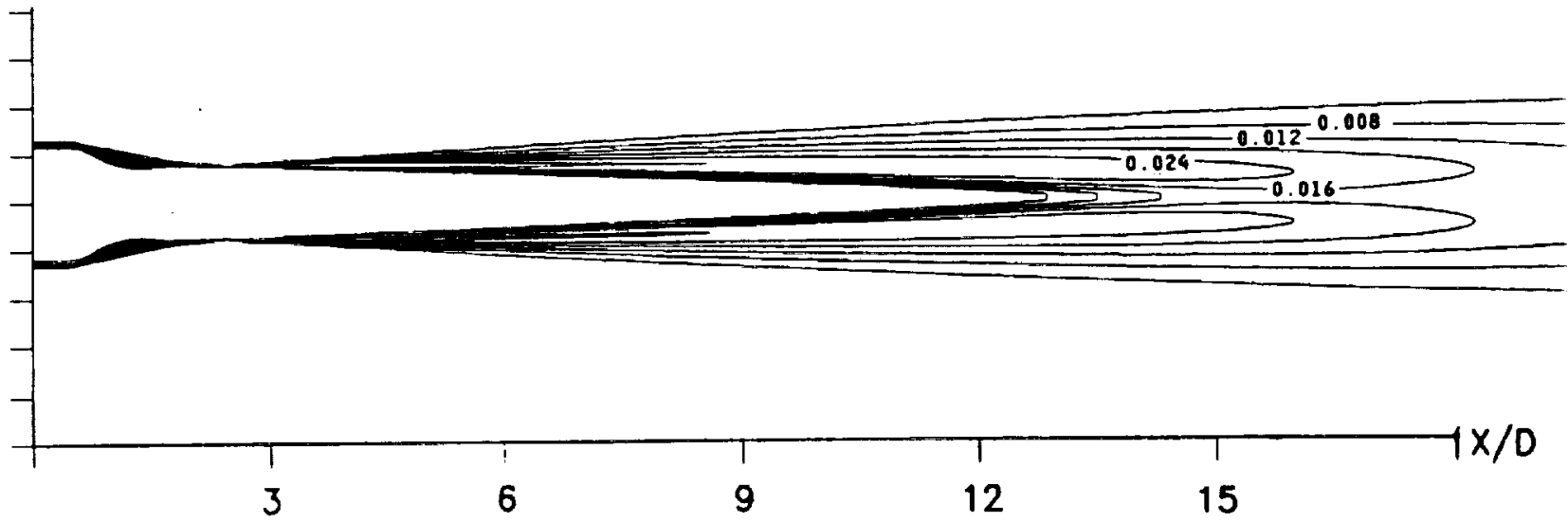

Fig. 5: Turbulent intensity contour plot (PARC-ke).
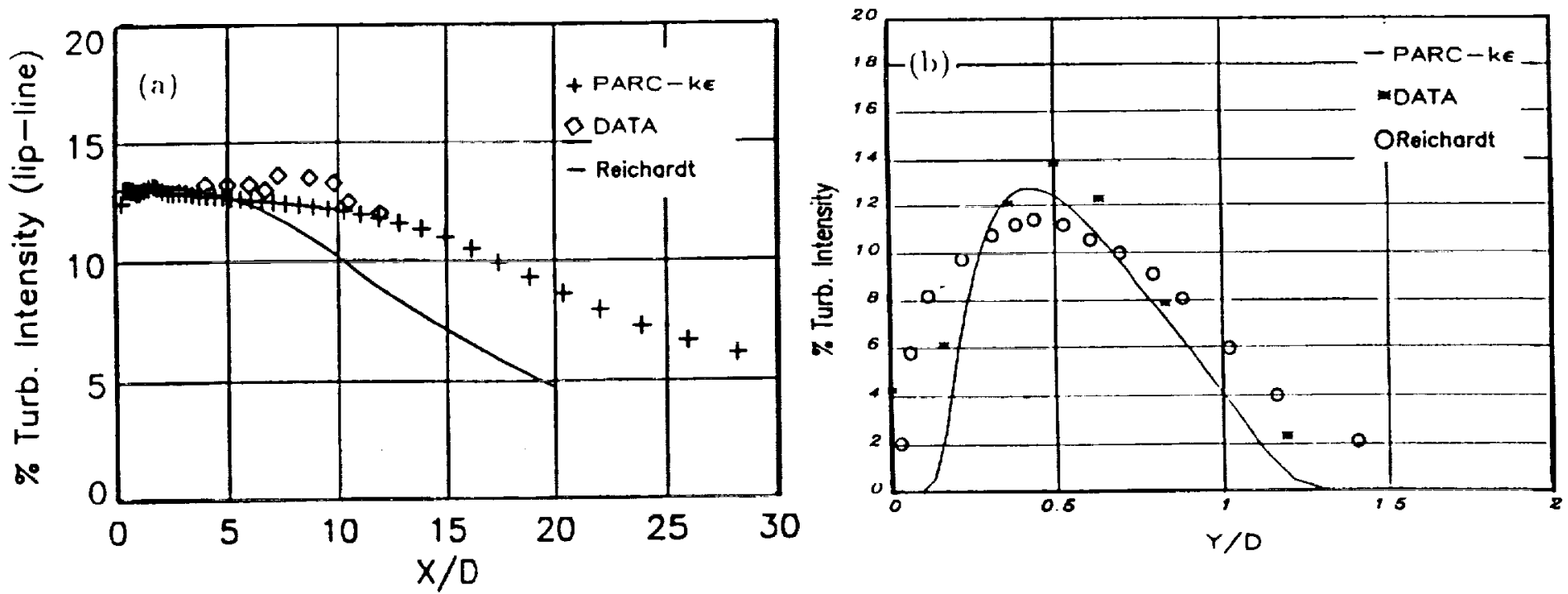

Fig. 6: Comparison of the turbulent intensity profiles with experimental results [1] (a) lip-line (b) $\mathrm{X} / D=8.21$. 

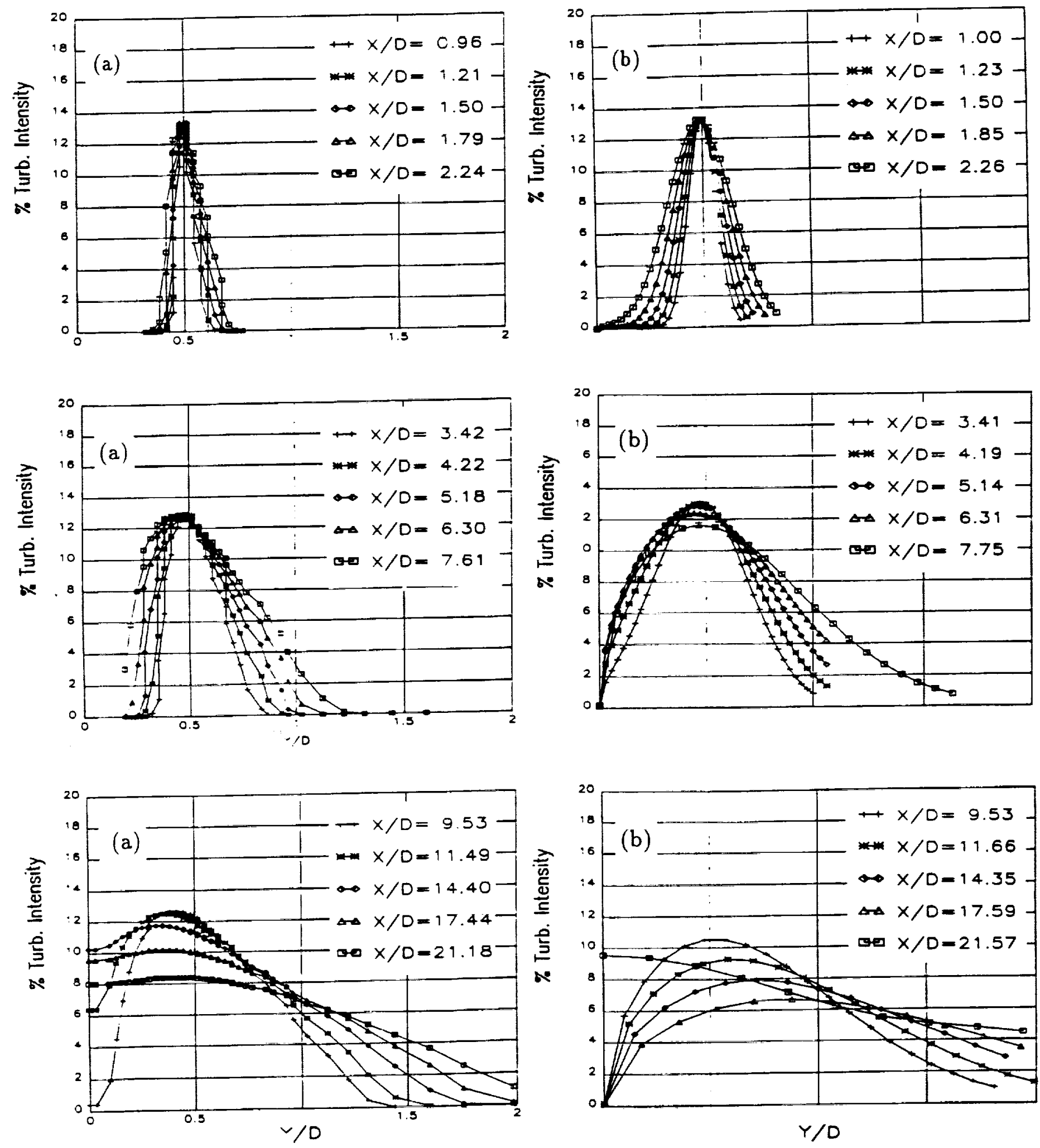

Fig. 7: Comparison of PARC-ke turbulent intensity profiles with Reichardt's solution at different axial locations, (a) PARC-ke (b) Reichardt's model. 

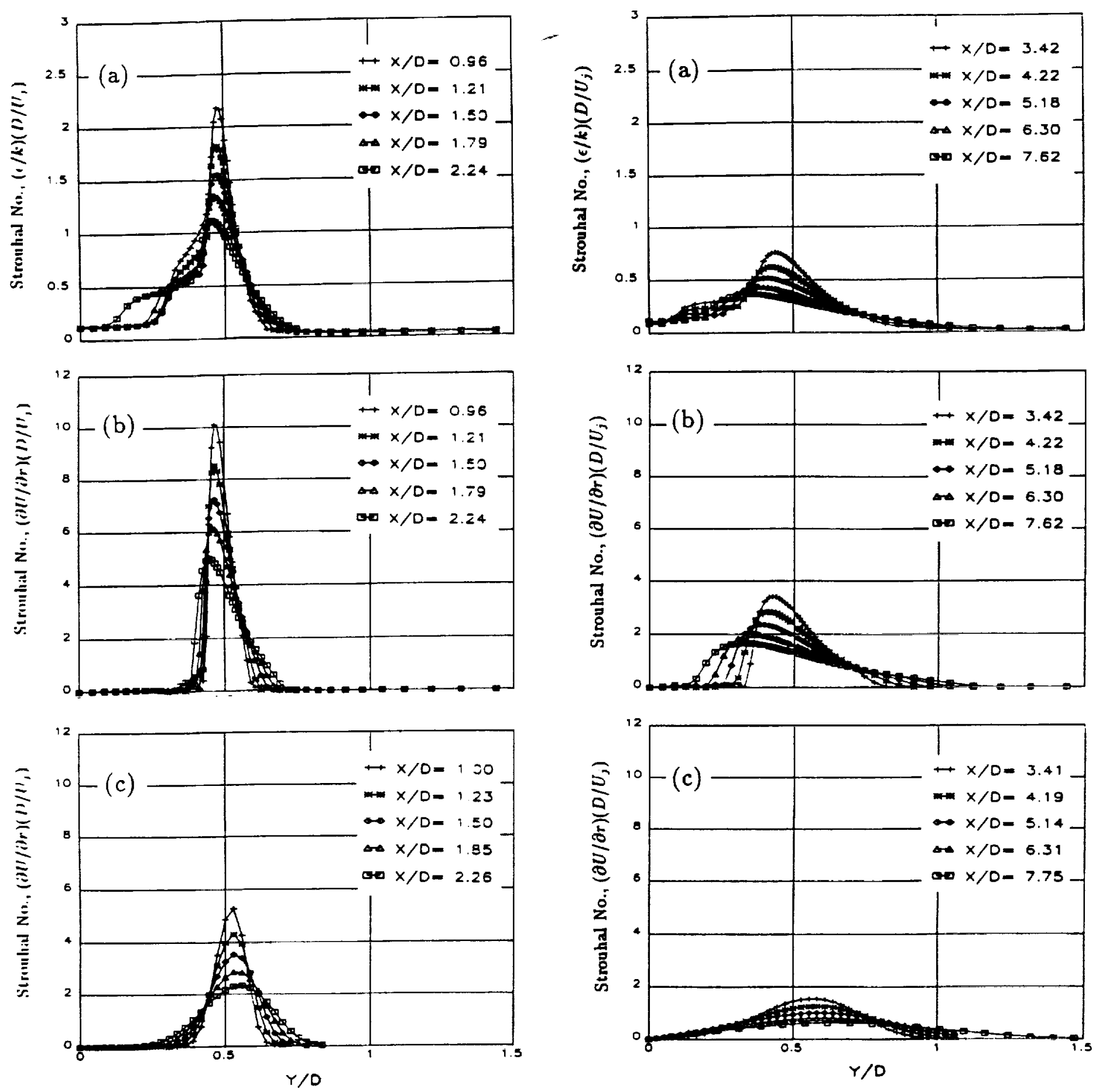

Fig. 8: Strouhal No., (a) St. defined as $(\epsilon / k)\left(D / U_{j}\right)$ obtained from PARC-ke. (b) St. defined as Fig. 9: Strouhal No., (a) and (b) obtained from PARC-ke, (c) Reichardt.

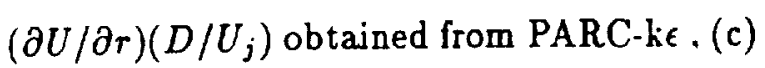
same as (b) but obtained from Reichardt's model. 


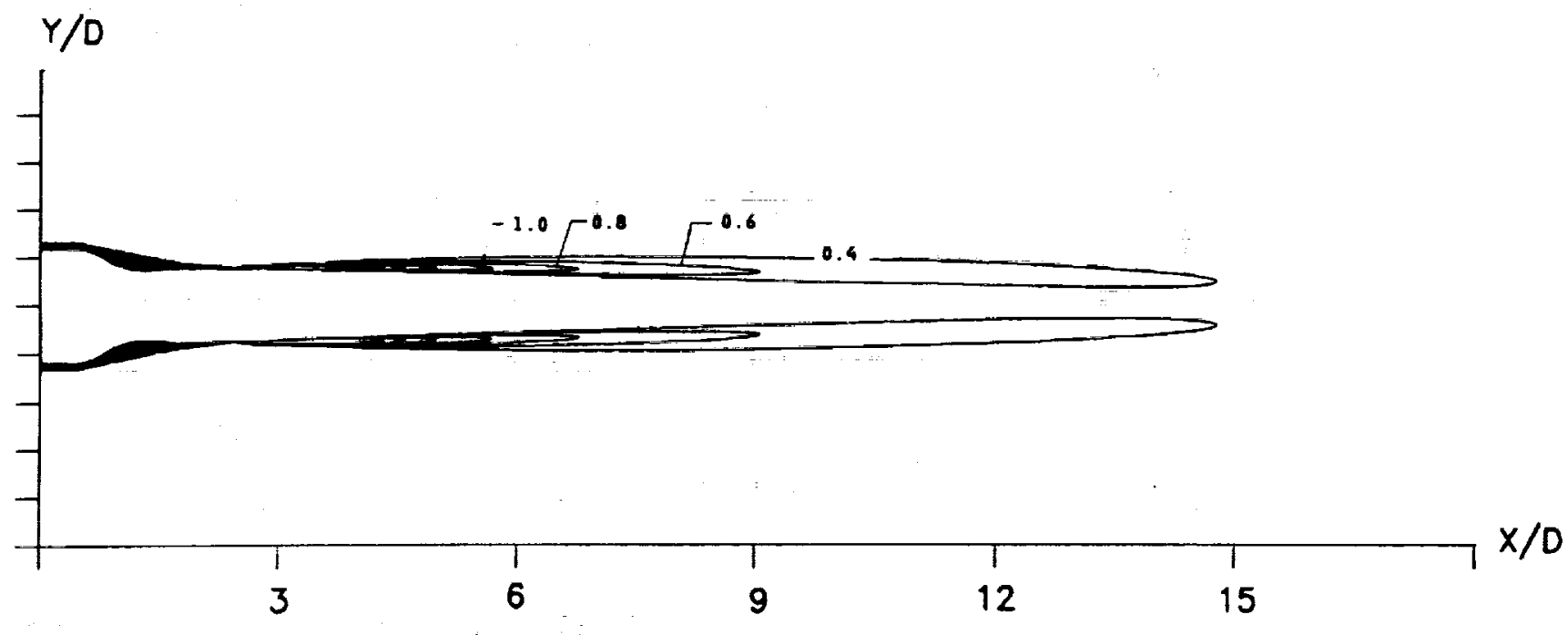

Fig. 10: Contour plot for dissipation rate of turbulent kinetic energy (PARC-ke).

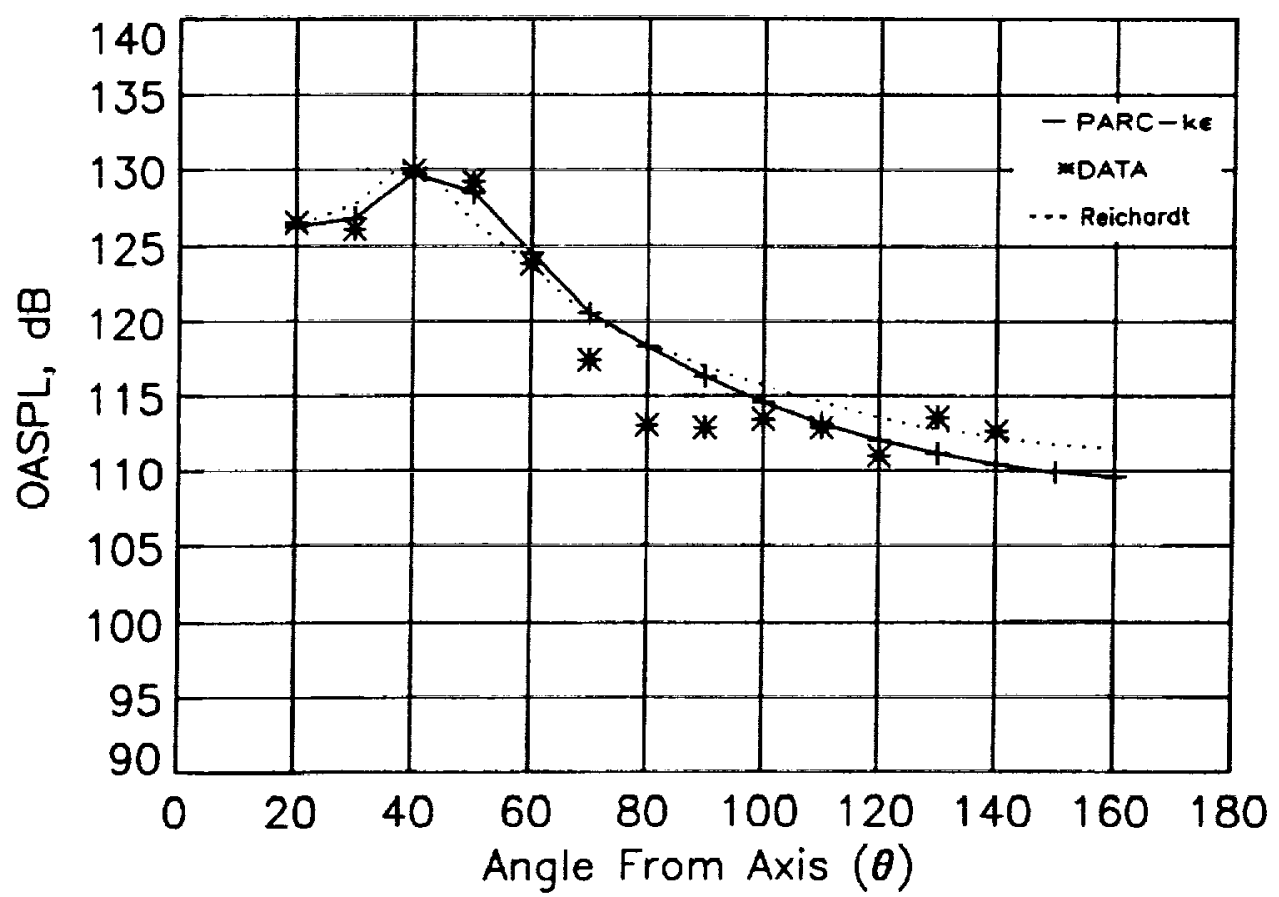

Fig. 11: Comparison of the overall sound pressure level directivity with data [1] on a 40 foot radius. 

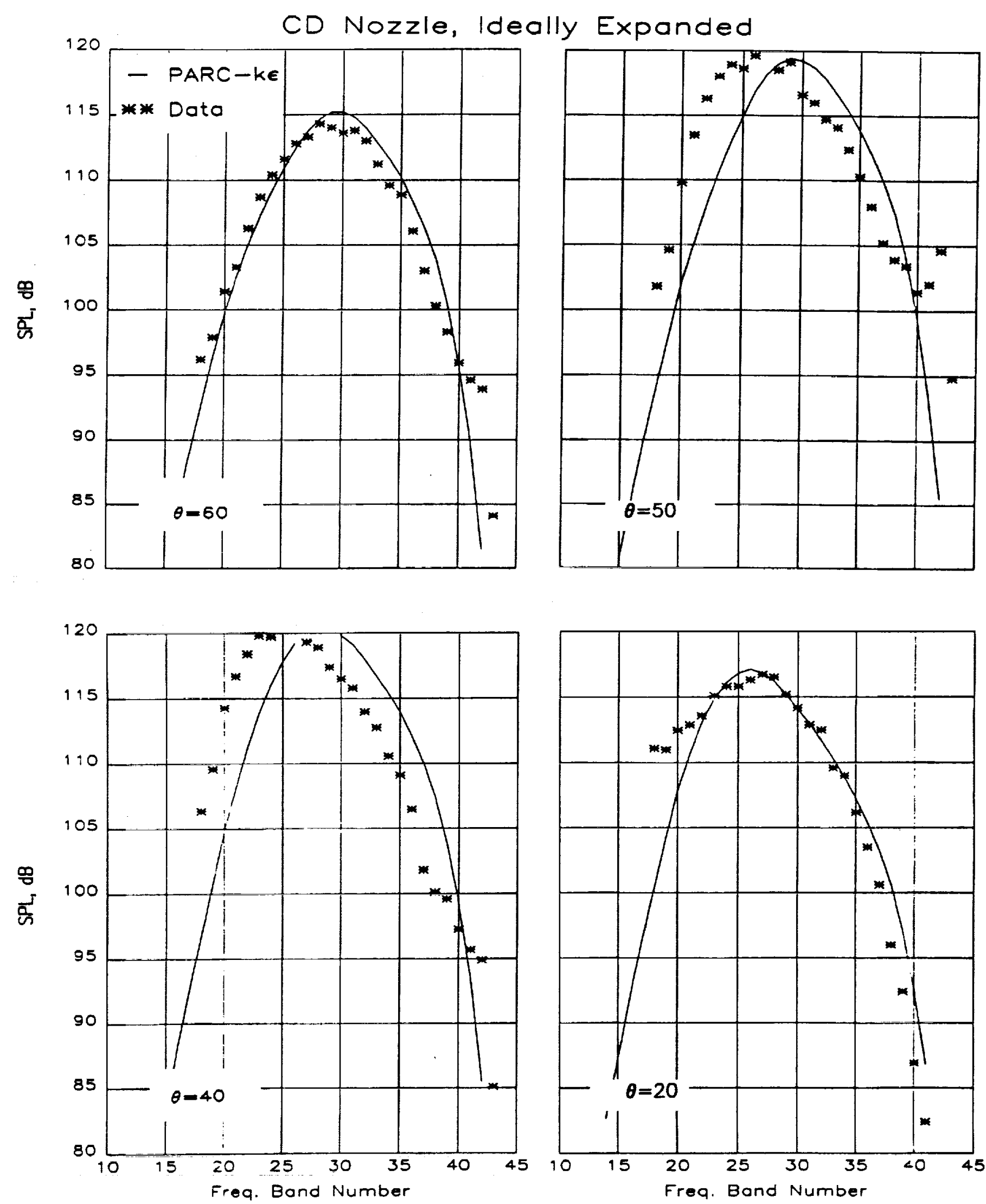

Fig. 12: Comparison of the spectral components of noise with data [1]. 


\section{REPORT DOCUMENTATION PAGE}

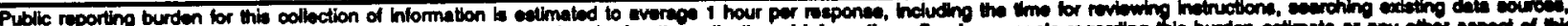

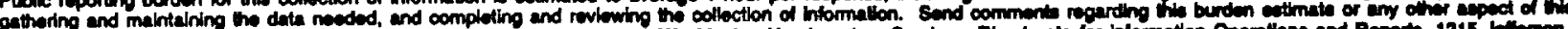

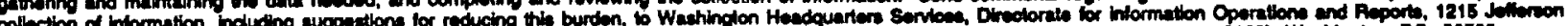

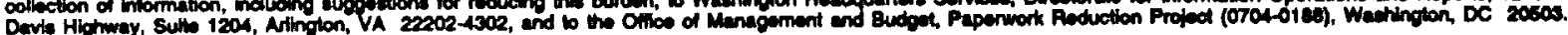

\begin{tabular}{|l|c|c|}
\hline 1. AOENCY USE ONLY (Leave blank) & $\begin{array}{c}\text { 2. REPOAT DATE } \\
1991\end{array}$ & $\begin{array}{r}\text { A. AEPOAT TYPE AND DATEC COVEREO } \\
\text { Technical Memorandum }\end{array}$
\end{tabular}

4. TILE AND \&UBTILE

Computation of Supersonic Jet Mixing Noise for an Axisymmetric CD

Nozzle Using k- $\varepsilon$ Turbulence Model

6. AUTHOR(3)

WU-537-02-23

Abbas Khavaran, Eugene A. Krejsa and Chan M. Kim

7. PERFORMINO OROANIZATION NAME(S) AND ADDRESS(ES)

- Pefformana organization REPORT NUMBER

National Aeronautics and Space Administration

Lewis Research Center

Cleveland, Ohio 44135-3191

E-6705

9. SPONSORINO/MONITORINO AGENCY NAMES(S) AND ADDRESS(ES)

10. SPONSORINOMMONTOAINO AOENCY REPORT MUMBER

National Aeronautics and Space Administration

Washington, D.C. 20546-0001

NASA TM-105338

AIAA -92-0500

11. SUPPLEMENTARY NOTES

Prepared for the 30th Aerospace Sciences Meeting and Exhibit sponsored by the American Institute of Aeronautics and Astronautics, Reno, Nevada, January 6-9, 1992. Abbas Khavaran, Sverdrup Technology, Inc., Lewis Research Center Group, 2001 Aerospace Parkway, Brook Park, Ohio 44142; Eugene A. Krejsa and Chan M. Kim, NASA Lewis Research Center. Responsible person, Abbas Khavaran, (216) 826-2293.

12.. DISTRIBUTION/AVAILABILITY STATEMENT

Unclassified - Unlimited

Subject Categories 71 and 07

12b. DISTAIBUTION CODE

\section{ABSTRACT (Mox/mum 200 words)}

The turbulent mixing noise of a supersonic jet is calculated for a round convergent-divergent nozzle at the design pressure ratio. Aerodynamic computations are performed using the PARC code with a k- $\varepsilon$ turbulence model. Lighthill's acoustic analogy combined with Ribner's assumption is adopted. The acoustics solution is based upon the methodology followed by GE in the MGB code. The source correlation function is expressed as a linear combination of second-order tensors. Assuming separable second-order correlations and incorporating Batchelor's isotropic turbulence model, the source term was calculated from the kinetic energy of turbulence. A Gaussian distribution for the time-delay of correlation was introduced. The CFD solution was used to obtain the source strength as well as the characteristic time-delay of correlation. The effect of sound/flow interaction was incorporated using the high frequency asymptotic solution to Lilley's equation for axisymmetric geometries. Acoustic results include sound pressure level directivity and spectra at different polar angles. The aerodynamic and acoustic results demonstrate favorable agreement with experimental data.

\begin{tabular}{|c|c|c|c|}
\hline \multirow{2}{*}{\multicolumn{3}{|c|}{$\begin{array}{l}\text { 14. SUBJECT TERMS } \\
\text { Jet aircraft noise; Noise prediction (aircraft); Acoustics; } \\
\text { Supersonic nozzles; Aerodynamic noise }\end{array}$}} & $\begin{array}{c}\text { 15. NUMBER OF PAOEB } \\
16\end{array}$ \\
\hline & & & $\begin{array}{r}\text { 16. PRICE CODE } \\
\text { A03 }\end{array}$ \\
\hline $\begin{array}{l}\text { 17. 8ECURITY CLASSIFICATION } \\
\text { OF REPORT } \\
\text { Unclassified }\end{array}$ & $\begin{array}{l}\text { 18. SECURITY CLASSIFICATION } \\
\text { OF THIS PAOE } \\
\text { Unclassified }\end{array}$ & $\begin{array}{l}\text { 19. SECURTY CLASSIFICATION } \\
\text { OF ABSTRACT } \\
\text { Unclassified }\end{array}$ & 20. LIMITATION OF ABSTRACT \\
\hline
\end{tabular}


